\title{
Some remarks on 'Multidimensional fixed point theorems for isotone mappings in partially ordered metric spaces'
}

Ravi P Agarwal ${ }^{1,2}$, Erdal Karapınar ${ }^{3,4}$ and Antonio-Francisco Roldán-López-de-Hierro ${ }^{5^{*}}$

"Correspondence:

afroldan@ujaen.es; aroldan@ugr.es ${ }^{5}$ Department of Mathematics,

University of Jaén, Campus Las Lagunillas, s/n, Jaén, 23071, Spain Full list of author information is available at the end of the article

\begin{abstract}
The main aim of this paper is to advise researchers in the field of Fixed Point Theory against an extended mistake that can be found in some proofs. We illustrate our claim proving that theorems in the very recent paper (Wang in Fixed Point Theory Appl. $2014: 137,2014)$ are incorrect, and we provide different corrected versions of them.
\end{abstract}

Keywords: fixed point; contractivity condition; isotone mapping; mixed monotone mapping

\section{Introduction and preliminaries}

Let $(X, d)$ be a metric space, let $k$ be a positive integer number, and let $X^{k}=X \times X \times \stackrel{(k)}{\cdots} \times X$ be the Cartesian product of $k$ identical copies of $X$. The function $\rho_{k}: X^{k} \times X^{k} \rightarrow[0, \infty)$ defined, for all $\left(y_{1}, y_{2}, \ldots, y_{k}\right),\left(v_{1}, v_{2}, \ldots, v_{k}\right) \in X^{k}$, by

$$
\rho_{k}\left(\left(y_{1}, y_{2}, \ldots, y_{k}\right),\left(v_{1}, v_{2}, \ldots, v_{k}\right)\right)=\frac{1}{k}\left[d\left(y_{1}, v_{1}\right)+d\left(y_{2}, v_{2}\right)+\cdots+d\left(y_{k}, v_{k}\right)\right]
$$

is a metric on $X^{k}$.

Let $\Phi$ denote the set of all continuous and strictly increasing functions $\phi:[0, \infty) \rightarrow$ $[0, \infty)$, and $\Psi$ denote the set of all functions $\psi:[0, \infty) \rightarrow[0, \infty)$ such that $\lim _{t \rightarrow r} \psi(t)>0$, for all $r>0$.

Inspired by Roldán et al.'s notion of a multidimensional fixed point (see [1-3]), Wang announced the following results in [4].

Theorem 1 (Wang [4, Theorem 3.1]) Let $(X, \preceq)$ be a partially ordered set and suppose that there is a metric $d$ on $X$ such that $(X, d)$ is a complete metric space. Let $T: X^{k} \rightarrow X^{k}$ be an isotone mapping for which there exist $\varphi \in \Phi$ and $\psi \in \Psi$ such that, for all $Y, V \in X^{k}$ with $Y \succeq V$,

$$
\varphi\left(\rho_{k}(T(Y), T(V))\right) \leq \varphi\left(\rho_{k}(Y, V)\right)-\psi\left(\rho_{k}(Y, V)\right),
$$

where $\rho_{k}$ is defined by (1). Suppose either

(a) $T$ is continuous or

(b) $(X, d, \preceq)$ is regular.

(02014 Agarwal et al.; licensee Springer. This is an Open Access article distributed under the terms of the Creative Commons Attribution License (http://creativecommons.org/licenses/by/2.0), which permits unrestricted use, distribution, and reproduction in any medium, provided the original work is properly cited. 
If there exists $Z_{0} \in X^{k}$ such that $Z_{0} \asymp T\left(Z_{0}\right)$, then $T$ has a fixed point.

As a consequence, she deduced the following result.

Theorem 2 (Wang [4, Corollary 3.2]) Let $(X, \preceq)$ be a partially ordered set and suppose that there is a metric $d$ on $X$ such that $(X, d)$ is a complete metric space. Let $T: X \rightarrow X$ be a nondecreasing mapping for which there exist $\varphi \in \Phi$ and $\psi \in \Psi$ such that, for all $y, v \in X$ with $y \succeq v$,

$$
\varphi(d(T(y), T(v))) \leq \varphi(d(y, v))-\psi(d(y, v)) .
$$

\section{Suppose either}

(a) $T$ is continuous or

(b) $(X, d, \preceq)$ is regular.

If there exists $z_{0} \in X$ such that $z_{0} \asymp T\left(z_{0}\right)$, then $T$ has a fixed point.

Remark 3 Theorems 1 and 2 are equivalent: Wang interpreted Theorem 2 as a corollary of Theorem 1 (taking $k=1$ ), but is also true that Theorem 1 is a particularization of Theorem 2 taking $\left(X^{k}, \rho_{k}, \preceq\right)$ rather than $(X, d, \preceq)$.

We claim that Theorem 2 is false, providing the following counterexample. As a consequence, all results in [4] are not correct.

\section{A counterexample}

Let $X=\mathbb{N}=\{1,2,3, \ldots\}$ be the set of all natural numbers endowed with the usual order $\leq$ of real numbers and the Euclidean metric $d(x, y)=|x-y|$, for all $x, y \in X$. Define $T: X \rightarrow X$ and $\phi, \psi:[0, \infty) \rightarrow[0, \infty)$ by

$$
\begin{aligned}
& T(x)=x+1, \quad \text { for all } x \in X ; \quad \varphi(t)=t, \quad \text { for all } t \in[0, \infty) ; \\
& \psi(t)= \begin{cases}0, & \text { if } t \text { is integer, } \\
1, & \text { otherwise }\end{cases}
\end{aligned}
$$

Clearly, $\varphi \in \Phi, \psi \in \Psi$ (in fact, notice that $\lim _{t \rightarrow r} \psi(t)=1$, for all $r \geq 0$ ). Furthermore, $T$ is a continuous, nondecreasing mapping. Moreover, it verifies condition (2) because if $y, v \in X=\mathbb{N}$ are natural numbers, then $d(y, v)$ is also a nonnegative integer number, so $\psi(d(y, v))=0$, and this proves that

$$
\begin{aligned}
\varphi(d(T(y), T(v))) & =d(y+1, v+1)=|(y+1)-(v+1)|=|y-v| \\
& =d(y, v)=\varphi(d(y, v))=\varphi(d(y, v))-\psi(d(y, v))
\end{aligned}
$$

(it is not necessary to assume that $y \succeq v$ ). Any point $z_{0} \in X$ verifies $z_{0} \preceq T\left(z_{0}\right)$. However, $T$ does not have any fixed point.

Remark 4 Notice that Wang's results are valid if we add the assumption $\psi(t)=0 \Leftrightarrow t=0$ but, in this case, the results are not as attractive. 


\section{Some considerations of Wang's paper}

We write the following in order to advise researchers against the mistake in Wang's proofs, which could be found on other papers.

In fixed point theory, given an operator $T: X \rightarrow X$, it is usual to consider a Picard sequence $x_{n+1}=T x_{n}$, for all $n \in \mathbb{N}$. In the context of partially ordered metric spaces, this sequence must be monotone (for instance, nondecreasing). Applying the contractivity condition (2), it follows that, for all $n \in \mathbb{N}$,

$$
\begin{aligned}
\varphi\left(d\left(x_{n+1}, x_{n+2}\right)\right) & =\varphi\left(d\left(T x_{n}, T x_{n+1}\right)\right) \leq \varphi\left(d\left(x_{n}, x_{n+1}\right)\right)-\psi\left(d\left(x_{n}, x_{n+1}\right)\right) \\
& \leq \varphi\left(d\left(x_{n}, x_{n+1}\right)\right) .
\end{aligned}
$$

Using the fact that $\varphi$ is strictly increasing, we have $d\left(x_{n+1}, x_{n+2}\right) \leq d\left(x_{n}, x_{n+1}\right)$, for all $n \in \mathbb{N}$, which means that $\left\{d\left(x_{n}, x_{n+1}\right)\right\}_{n \in \mathbb{N}}$ is a nonincreasing sequence of nonnegative real numbers. As a consequence, it is convergent. Let $L \geq 0$ be its limit. In order to prove that $L=0$, we reason by contradiction assuming that $L>0$. Taking into account that

$$
\varphi\left(d\left(x_{n+1}, x_{n+2}\right)\right) \leq \varphi\left(d\left(x_{n}, x_{n+1}\right)\right)-\psi\left(d\left(x_{n}, x_{n+1}\right)\right), \quad \text { for all } n \in \mathbb{N}
$$

it is usual to take the limit in the previous inequality. As $\varphi$ is continuous and $\lim _{t \rightarrow L} \psi(t)>0$ by hypothesis, the author tried to deduce that

$$
\begin{aligned}
\varphi(L) & =\lim _{n \rightarrow \infty} \varphi\left(d\left(x_{n+1}, x_{n+2}\right)\right) \leq \lim _{n \rightarrow \infty}\left[\varphi\left(d\left(x_{n}, x_{n+1}\right)\right)-\psi\left(d\left(x_{n}, x_{n+1}\right)\right)\right] \\
& =\lim _{n \rightarrow \infty} \varphi\left(d\left(x_{n}, x_{n+1}\right)\right)-\lim _{n \rightarrow \infty} \psi\left(d\left(x_{n}, x_{n+1}\right)\right) \\
& =\varphi(L)-\lim _{t \rightarrow L} \psi(t)<\varphi(L),
\end{aligned}
$$

which is a contradiction. However, the equality

$$
\lim _{n \rightarrow \infty} \psi\left(d\left(x_{n}, x_{n+1}\right)\right)=\lim _{t \rightarrow L} \psi(t)
$$

can be false: as in the previous counterexample, the sequence $\left\{\psi\left(d\left(x_{n}, x_{n+1}\right)\right)\right\}_{n \in \mathbb{N}}$ can be identically zero but the limit $\lim _{t \rightarrow L} \psi(t)$ must take a positive value.

\section{A suggestion to correct Wang's paper}

In this section, inspired by Wang's paper [4], we suggest a new theorem in the context of partially ordered metric spaces. We also underline that one can easily state the same theorem in the frame of multidimensional fixed points. In fact, they are equivalent as is mentioned in Section 1.

Firstly, we give a modified version of the collection of auxiliary functions $\Psi$ in the following way. Let $\Psi^{\prime}$ denote the set of all lower semi-continuous functions $\psi:[0, \infty) \rightarrow[0, \infty)$ such that $\psi(t)=0 \Leftrightarrow t=0$. The following two lemmas will be useful in the proofs of the upcoming theorems.

Lemma 5 (See, e.g., [5]) Let $(X, d)$ be a metric space and let $\left\{x_{n}\right\}$ be a sequence in $X$ such that

$$
\lim _{n \rightarrow \infty} d\left(x_{n}, x_{n+1}\right)=0 .
$$


If $\left\{x_{n}\right\}$ is not a Cauchy sequence in $(X, d)$, then there exist $\varepsilon>0$ and two sequences $\{n(k)\}$ and $\{m(k)\}$ of positive integers such that, for all $k \in \mathbb{N}$,

$$
k \leq n(k)<m(k) \quad \text { and } \quad d\left(x_{n(k)}, x_{m(k)-1}\right) \leq \varepsilon<d\left(x_{n(k)}, x_{m(k)}\right) .
$$

Furthermore,

$$
\begin{aligned}
\lim _{k \rightarrow \infty} d\left(x_{n(k)}, x_{m(k)}\right) & =\lim _{k \rightarrow \infty} d\left(x_{n(k)+1}, x_{m(k)}\right) \\
& =\lim _{k \rightarrow \infty} d\left(x_{n(k)}, x_{m(k)+1}\right) \\
& =\lim _{k \rightarrow \infty} d\left(x_{n(k)+1}, x_{m(k)+1}\right)=\varepsilon
\end{aligned}
$$

Lemma 6 Let $\varphi \in \Phi, \psi \in \Psi^{\prime}$, and let $\left\{t_{n}\right\},\left\{s_{n}\right\},\left\{a_{n}\right\} \subset[0, \infty)$ be three sequences of nonnegative real numbers such that

$$
\varphi\left(t_{n}\right) \leq \varphi\left(s_{n}\right)-\psi\left(s_{n}\right)+a_{n}, \quad \text { for all } n \in \mathbb{N} .
$$

If there exists $\alpha \in[0, \infty)$ such that $\left\{t_{n}\right\} \rightarrow \alpha,\left\{s_{n}\right\} \rightarrow \alpha,\left\{a_{n}\right\} \rightarrow 0$, and $s_{n}>\alpha$, for all $n \in \mathbb{N}$, then $\alpha=0$.

Proof Notice that

$$
0 \leq \psi\left(s_{n}\right) \leq \varphi\left(s_{n}\right)-\varphi\left(t_{n}\right)+a_{n}, \quad \text { for all } n \in \mathbb{N} .
$$

As $\left\{t_{n}\right\} \rightarrow \alpha,\left\{s_{n}\right\} \rightarrow \alpha,\left\{a_{n}\right\} \rightarrow 0$, and $\psi$ is continuous, we deduce that

$$
\lim _{n \rightarrow \infty} \psi\left(s_{n}\right)=0
$$

As $s_{n}>\alpha$, for all $n \in \mathbb{N}$, and $\psi$ is lower semi-continuous, we deduce that

$$
0 \leq \psi(\alpha) \leq \liminf _{t \rightarrow \alpha^{+}} \psi(t)=\liminf _{n \rightarrow \infty} \psi\left(s_{n}\right)=\lim _{n \rightarrow \infty} \psi\left(s_{n}\right)=0 .
$$

Therefore, $\psi(\alpha)=0$. As $\psi \in \Psi^{\prime}$, we conclude that $\alpha=0$.

Theorem 7 Let $(X, \preceq)$ be a partially ordered set and suppose that there is a metric $d$ on $X$ such that $(X, d)$ is a complete metric space. Let $T: X \rightarrow X$ be a nondecreasing mapping for which there exist $\varphi \in \Phi, \psi \in \Psi^{\prime}$ and $L \geq 0$ such that

$$
\varphi(d(T x, T y)) \leq \varphi\left(M_{d}(x, y)\right)-\psi\left(M_{d}(x, y)\right)+L N_{d}(x, y),
$$

for all $x, y \in X$ with $x \preceq y$, where

$$
M_{d}(x, y)=\max \left\{d(x, y), d(x, T x), d(y, T y), \frac{d(y, T x)+d(x, T y)}{2}\right\}
$$

and

$$
N_{d}(x, y)=\min \{d(x, T x), d(y, T y), d(x, T y), d(y, T x)\} .
$$


Suppose either

(a) $T$ is continuous or

(b) $(X, d, \preceq)$ is regular.

If there exists $z_{0} \in X$ such that $z_{0} \asymp T\left(z_{0}\right)$, then $T$ has a fixed point.

Proof By assumption, there exists $z_{0} \in X$ such that $z_{0} \asymp T\left(z_{0}\right)$. Without loss of generality, we assume that $z_{0} \preceq T z_{0}$ (the case $z_{0} \succeq T z_{0}$ can be treated analogously). Define an iterative sequence $\left\{z_{n}\right\}$ as $z_{n+1}=T z_{n}$ for each $n \in\{0,1,2, \ldots\}$. If $z_{n_{0}}=T z_{n_{0}}$ for some $n_{0}$, then we conclude the assertion of the theorem. So, assume that $z_{n+1} \neq z_{n}$ for each $n \in\{0,1,2, \ldots\}$. Since $T$ is nondecreasing, $z_{0} \preceq T z_{0}=z_{1}$ implies that $z_{1}=T z_{0} \preceq T z_{1}=z_{2}$. Recursively, we derive that

$$
z_{n} \preceq z_{n+1}, \quad \text { for each } n \in\{0,1,2, \ldots\} \text {. }
$$

Letting $x=z_{n}$ and $y=z_{n+1}$ in (3) we get, for all $n \in \mathbb{N}$,

$$
\begin{aligned}
\varphi\left(d\left(z_{n+1}, z_{n+2}\right)\right) & =\varphi\left(d\left(T z_{n}, T z_{n+1}\right)\right) \\
& \leq \varphi\left(M_{d}\left(z_{n}, z_{n+1}\right)\right)-\psi\left(M_{d}\left(z_{n}, z_{n+1}\right)\right)+L N_{d}\left(z_{n}, z_{n+1}\right) .
\end{aligned}
$$

By elementary calculation we find that $N_{d}\left(z_{n}, z_{n+1}\right)=0$, and

$$
M_{d}\left(z_{n}, z_{n+1}\right)=\max \left\{d\left(z_{n}, z_{n+1}\right), d\left(z_{n+1}, z_{n+2}\right)\right\} .
$$

If there exists some $n$ such that $M_{d}\left(z_{n}, z_{n+1}\right)=d\left(z_{n+1}, z_{n+2}\right)$, then (4) turns into

$$
\varphi\left(d\left(z_{n+1}, z_{n+2}\right)\right) \leq \varphi\left(d\left(z_{n+1}, z_{n+2}\right)\right)-\psi\left(d\left(z_{n+1}, z_{n+2}\right)\right)
$$

which is a contradiction because $d\left(z_{n+1}, z_{n+2}\right)>0$. Hence, (4) is equivalent to

$$
\begin{aligned}
\varphi\left(d\left(z_{n+1}, z_{n+2}\right)\right) & \leq \varphi\left(d\left(z_{n}, z_{n+1}\right)\right)-\psi\left(d\left(z_{n}, z_{n+1}\right)\right) \\
& <\varphi\left(d\left(z_{n}, z_{n+1}\right)\right)
\end{aligned}
$$

for all $n \in \mathbb{N}$. Since $\varphi$ is a strictly increasing function, we have, for all $n \in \mathbb{N}$,

$$
d\left(z_{n+1}, z_{n+2}\right)<d\left(z_{n}, z_{n+1}\right) .
$$

Thus, $\left\{d\left(z_{n}, z_{n+1}\right)\right\}$ is a decreasing sequence that is bounded from below. Thus, there exists $\delta \geq 0$ such that $\lim _{n \rightarrow \infty} d\left(z_{n}, z_{n+1}\right)=\delta$. We assert that $\delta=0$. Suppose, on the contrary, that $\delta>0$. Letting $n \rightarrow \infty$ in (5) and regarding the properties of the auxiliary functions $\varphi, \phi$, we derive that

$$
\varphi(\delta) \leq \varphi(\delta)-\psi(\delta)
$$

Hence, $\psi(\delta)=0$ and, as a result, $\delta=0$. Thus,

$$
\lim _{n \rightarrow \infty} d\left(z_{n}, z_{n+1}\right)=0
$$


In what follows, we prove that $\left\{z_{n}\right\}$ is a Cauchy sequence. Suppose, on the contrary, that $\left\{z_{n}\right\}$ is not Cauchy. By Lemma 5 , there exist $\varepsilon>0$ and two sequences $\{n(k)\}$ and $\{m(k)\}$ of positive integers such that, for all $k \in \mathbb{N}$,

$$
\begin{aligned}
& k \leq n(k)<m(k), \\
& d\left(z_{n(k)}, z_{m(k)-1}\right) \leq \varepsilon<d\left(z_{n(k)}, z_{m(k)}\right)
\end{aligned}
$$

and

$$
\begin{aligned}
\lim _{k \rightarrow \infty} d\left(z_{n(k)}, z_{m(k)}\right) & =\lim _{k \rightarrow \infty} d\left(z_{n(k)+1}, z_{m(k)}\right) \\
& =\lim _{k \rightarrow \infty} d\left(z_{n(k)}, z_{m(k)+1}\right) \\
& =\lim _{k \rightarrow \infty} d\left(z_{n(k)+1}, z_{m(k)+1}\right)=\varepsilon .
\end{aligned}
$$

As $\left\{z_{n}\right\}$ is nondecreasing, $z_{n(k)} \preccurlyeq z_{m(k)}$, for all $k \in \mathbb{N}$. Applying the contractivity condition (3), it follows, for all $k \in \mathbb{N}$, that

$$
\begin{aligned}
\varphi\left(d\left(z_{n(k)+1}, z_{m(k)+1}\right)\right)= & \varphi\left(d\left(T z_{n(k)}, T z_{m(k)}\right)\right) \\
\leq & \varphi\left(M_{d}\left(z_{n(k)}, z_{m(k)}\right)\right)-\psi\left(M_{d}\left(z_{n(k)}, z_{m(k)}\right)\right) \\
& +L N_{d}\left(z_{n(k)}, z_{m(k)}\right),
\end{aligned}
$$

where

$$
\begin{aligned}
M_{d}\left(z_{n(k)}, z_{m(k)}\right)= & \max \left\{d\left(z_{n(k)}, z_{m(k)}\right), d\left(z_{n(k)}, z_{n(k)+1}\right),\right. \\
& \left.d\left(z_{m(k)}, z_{m(k)+1}\right), \frac{d\left(z_{m(k)}, z_{n(k)+1}\right)+d\left(z_{n(k)}, z_{m(k)+1}\right)}{2}\right\}
\end{aligned}
$$

and

$$
\begin{aligned}
N_{d}\left(z_{n(k)}, z_{m(k)}\right)= & \min \left\{d\left(z_{n(k)}, z_{n(k)+1}\right), d\left(z_{m(k)}, z_{m(k)+1}\right),\right. \\
& \left.d\left(z_{n(k)}, z_{m(k)+1}\right), d\left(z_{m(k)}, z_{n(k)+1}\right)\right\} .
\end{aligned}
$$

As $\left\{d\left(z_{n(k)}, z_{n(k)+1}\right)\right\}_{k \in \mathbb{N}} \rightarrow 0$,

$$
\lim _{k \rightarrow \infty} N_{d}\left(z_{n(k)}, z_{m(k)}\right)=0 .
$$

Furthermore, by (6), we have

$$
\lim _{k \rightarrow \infty} M_{d}\left(z_{n(k)}, z_{m(k)}\right)=\max \{\varepsilon, 0,0, \varepsilon\}=\varepsilon .
$$

Using the sequences

$$
\begin{aligned}
& \left\{t_{k}=d\left(z_{n(k)+1}, z_{m(k)+1}\right)\right\}_{k \in \mathbb{N}}, \quad\left\{s_{k}=M_{d}\left(z_{n(k)}, z_{m(k)}\right)\right\}_{k \in \mathbb{N}} \quad \text { and } \\
& \left\{a_{k}=L N_{d}\left(z_{n(k)}, z_{m(k)}\right)\right\}_{k \in \mathbb{N}},
\end{aligned}
$$


Lemma 6 guarantees that $\varepsilon=\alpha=0$, which is a contradiction. As a consequence, we must admit that $\left\{z_{n}\right\}$ is a Cauchy sequence in $(X, d)$. As it is complete, there exists $z \in X$ such that

$$
\lim _{n \rightarrow \infty} z_{n}=z
$$

If $T$ is continuous, it is clear that $z$ is a fixed point of $T$.

Suppose that condition (b) holds. Hence, as $\left\{z_{n}\right\}$ is nondecreasing and converges to $z$, we have $z_{n} \preceq z$, for all $n=\{0,1,2, \ldots\}$. Due to (3), we have, for all $n \in \mathbb{N}$,

$$
\varphi\left(d\left(z_{n+1}, T z\right)\right) \leq \varphi\left(M_{d}\left(z_{n}, z\right)\right)-\psi\left(M_{d}\left(z_{n}, z\right)\right)+L N_{d}\left(z_{n}, z\right)
$$

where

$$
M_{d}\left(z_{n}, z\right)=\max \left\{d\left(z_{n}, z\right), d\left(z_{n}, z_{n+1}\right), d(z, T z), \frac{d\left(z, z_{n+1}\right)+d\left(z_{n}, T z\right)}{2}\right\}
$$

and

$$
N_{d}\left(z_{n}, z\right)=\min \left\{d\left(z_{n}, z_{n+1}\right), d(z, T z), d\left(z, z_{n+1}\right), d\left(z_{n}, T z\right)\right\} .
$$

Letting $n \rightarrow \infty$ in (7), we get

$$
\varphi(d(z, T z)) \leq \varphi(d(z, T z))-\psi(d(z, T z)) \leq \varphi(d(z, T z))
$$

so $\psi(d(z, T z))=0$ and $d(z, T z)=0$. As a consequence, $T z=z$, which completes the proof.

The following corollary follows from Theorem 7 using $L=0$.

Corollary 8 Let $(X, \preceq)$ be a partially ordered set and suppose that there is a metric $d$ on $X$ such that $(X, d)$ is a complete metric space. Let $T: X \rightarrow X$ be a nondecreasing mapping for which there exist $\varphi \in \Phi, \psi \in \Psi^{\prime}$ such that

$$
\varphi(d(T x, T y)) \leq \varphi\left(M_{d}(x, y)\right)-\psi\left(M_{d}(x, y)\right)
$$

for all $x, y \in X$ with $x \preceq y$, where

$$
M_{d}(x, y)=\max \left\{d(x, y), d(x, T x), d(y, T y), \frac{d(y, T x)+d(x, T y)}{2}\right\} .
$$

\section{Suppose either}

(a) $T$ is continuous or

(b) $(X, d, \preceq)$ is regular.

If there exists $z_{0} \in X$ such that $z_{0} \asymp T\left(z_{0}\right)$, then $T$ has a fixed point.

In the next result, we take $\varphi$ as the identity mapping on $[0, \infty)$, which clearly belongs to $\Phi$. 
Corollary 9 Let $(X, \preceq)$ be a partially ordered set and suppose that there is a metric $d$ on $X$ such that $(X, d)$ is a complete metric space. Let $T: X \rightarrow X$ be a nondecreasing mapping for which there exists $\psi \in \Psi^{\prime}$ such that

$$
d(T x, T y) \leq M_{d}(x, y)-\psi\left(M_{d}(x, y)\right)
$$

for all $x, y \in X$ with $x \preceq y$, where

$$
M_{d}(x, y)=\max \left\{d(x, y), d(x, T x), d(y, T y), \frac{d(y, T x)+d(x, T y)}{2}\right\} .
$$

Suppose either

(a) $T$ is continuous or

(b) $(X, d, \preceq)$ is regular.

If there exists $z_{0} \in X$ such that $z_{0} \asymp T\left(z_{0}\right)$, then $T$ has a fixed point.

The following result is not a direct consequence of Theorem 7, but its proof is verbatim the proof of Theorem 7. Thus, we skip it.

Theorem 10 Let $(X, \preceq)$ be a partially ordered set and suppose that there is a metric $d$ on $X$ such that $(X, d)$ is a complete metric space. Let $T: X \rightarrow X$ be a nondecreasing mapping for which there exist $\varphi \in \Phi, \psi \in \Psi^{\prime}$, and $L \geq 0$ such that

$$
\varphi(d(T x, T y)) \leq \varphi(d(x, y))-\psi(d(x, y))+L N_{d}(x, y)
$$

for all $x, y \in X$ with $x \preceq y$, where

$$
N_{d}(x, y)=\min \{d(x, T x), d(y, T y), d(x, T y), d(y, T x)\} .
$$

Suppose either

(a) $T$ is continuous or

(b) $(X, d, \preceq)$ is regular.

If there exists $z_{0} \in X$ such that $z_{0} \asymp T\left(z_{0}\right)$, then $T$ has a fixed point.

If $L=0$ in the previous theorem, we obtain the following result.

Corollary 11 Let $(X, \preceq)$ be a partially ordered set and suppose that there is a metric $d$ on $X$ such that $(X, d)$ is a complete metric space. Let $T: X \rightarrow X$ be a nondecreasing mapping for which there exist $\varphi \in \Phi, \psi \in \Psi^{\prime}$ such that

$$
\varphi(d(T x, T y)) \leq \varphi(d(x, y))-\psi(d(x, y))
$$

for all $x, y \in X$ with $x \preceq y$. Suppose either
(a) $T$ is continuous or
(b) $(X, d, \preceq)$ is regular.

If there exists $z_{0} \in X$ such that $z_{0} \asymp T\left(z_{0}\right)$, then $T$ has a fixed point.

If we take $\varphi$ as the identity mapping on $[0, \infty)$, we derive the following result. 
Corollary 12 Let $(X, \preceq)$ be a partially ordered set and suppose that there is a metric $d$ on $X$ such that $(X, d)$ is a complete metric space. Let $T: X \rightarrow X$ be a nondecreasing mapping for which there exists $\psi \in \Psi^{\prime}$ such that

$$
d(T x, T y) \leq d(x, y)-\psi(d(x, y))
$$

for all $x, y \in X$ with $x \preceq y$. Suppose either

(a) $T$ is continuous or

(b) $(X, d, \preceq)$ is regular.

If there exists $z_{0} \in X$ such that $z_{0} \asymp T\left(z_{0}\right)$, then $T$ has a fixed point.

In the sequel we suggest another theorem by changing the properties of the auxiliary functions in the following way.

Let $\Psi^{\prime \prime}$ denote the set of all functions $\psi:[0, \infty) \rightarrow[0, \infty)$ such that

1. $\psi(t)=0 \Leftrightarrow t=0$ and $\liminf _{n \rightarrow \infty} \psi\left(t_{n}\right)>0$ whenever $\lim _{n \rightarrow \infty} t_{n}=t>0$.

2. $\psi(t)>\varphi(t)-\varphi(t-)$ for any $t>0$, where $\varphi(t-)$ is the left limit of $\varphi$ at $t$, where $\varphi \in \Phi$.

Theorem 13 Let $(X, \preceq)$ be a partially ordered set and suppose that there is a metric $d$ on $X$ such that $(X, d)$ is a complete metric space. Let $T: X \rightarrow X$ be a nondecreasing mapping for which there exist $\varphi \in \Phi$ and $\psi \in \Psi^{\prime \prime}, L \geq 0$ such that

$$
\varphi(d(T y, T v)) \leq \varphi\left(M_{d}(y, v)\right)-\psi\left(M_{d}(y, v)\right)+L N_{d}(y, v)
$$

for all $y, v \in X$ with $y \preceq v$, where

$$
M_{d}(y, v)=\max \left\{d(y, v), d(y, T y), d(v, T v), \frac{d(y, T v)+d(v, T y)}{2}\right\}
$$

and

$$
N_{d}(y, v)=\min \{d(y, T y), d(v, T v), d(v, T y), d(y, T v)\} .
$$

Suppose either

(a) $T$ is continuous or

(b) $(X, d, \preceq)$ is regular.

If there exists $z_{0} \in X$ such that $z_{0} \asymp T\left(z_{0}\right)$, then $T$ has a fixed point.

The proof is analog to the proof of Theorem 7 and, hence, we skip it.

Remark 14 As in Theorem 13, by changing the property of the auxiliary function, we get various results (see, e.g., [6-9] and related references therein).

\section{Fixed point theorems from (one dimensional) fixed point to multidimensional fixed point}

As discussed in Remark 3, multidimensional fixed point theorems are equivalent to (one dimensional) fixed point theorems. Thus, Theorem 7 can be translated in a multidimensional case as follows. 
Theorem 15 Let $(X, \preceq)$ be a partially ordered set and suppose that there is a metric $d$ on $X$ such that $(X, d)$ is a complete metric space. Let $T: X^{k} \rightarrow X^{k}$ be a nondecreasing mapping for which there exist $\varphi \in \Phi, \psi \in \Psi^{\prime}$, and $L \geq 0$ such that

$$
\varphi\left(\rho_{k}(T V, T Y)\right) \leq \varphi\left(M_{\rho_{k}}(V, Y)\right)-\psi\left(M_{\rho_{k}}(V, Y)\right)+L N_{\rho_{k}}(V, Y)
$$

for all $V, Y \in X$ with $V \preceq Y$, where

$$
M_{\rho_{k}}(V, Y)=\max \left\{\rho_{k}(V, Y), \rho_{k}(V, T V), \rho_{k}(Y, T Y), \frac{\rho_{k}(Y, T V)+\rho_{k}(V, T Y)}{2}\right\}
$$

and

$$
N_{\rho_{k}}(V, Y)=\min \left\{\rho_{k}(V, T V), \rho_{k}(Y, T Y), \rho_{k}(V, T Y), \rho_{k}(Y, T V)\right\}
$$

Suppose either

(a) $T$ is continuous or

(b) $(X, d, \preceq)$ is regular.

If there exists $Z_{0} \in X^{k}$ such that $Z_{0} \asymp T\left(Z_{0}\right)$, then $T$ has a fixed point.

In similar way, we may state the analog of Corollary 8 , Corollary 9, Theorem 10, Corollary 11, Corollary 12.

\section{Applications}

In this section, based on the results in [10], we propose an application to our results. Consider the integral equation

$$
u(t)=\int_{0}^{T} K(t, s, u(s)) d s+g(t), \quad t \in[0, T]
$$

where $T>0$. We introduce the following space:

$$
C[0, T]=\{u:[0, T] \rightarrow \mathbb{R}: u \text { is continuous on }[0, T]\}
$$

equipped with the metric

$$
d(u, v)=\sup _{t \in[0, T]}|u(t)-v(t)|, \quad \text { for each } u, v \in C[0, T] .
$$

It is clear that $(C[0, T], d)$ is a complete metric space. Furthermore, $C[0, T]$ can be equipped with the partial order $\preceq$ as follows:

$$
u, v \in C[0, T], \quad u \preceq v \quad \Leftrightarrow \quad u(t) \leq v(t), \quad \text { for each } t \in[0, T] .
$$

Due to [11], we know that $(C[0, T], d, \preceq)$ is regular.

In what follows we state the main result of this section.

Theorem 16 We assume that the following hypotheses hold: 
(i) $K:[0, T] \times[0, T] \times \mathbb{R} \rightarrow \mathbb{R}$ and $g: \mathbb{R} \rightarrow \mathbb{R}$ are continuous,

(ii) for all $s, t \in[0, T]$ and $u, v \in C[0, T]$ with $v \preceq u$, we have

$$
K(t, s, v(s)) \leq K(t, s, u(s))
$$

(iii) there exists a continuous function $G:[0, T] \times[0, T] \rightarrow[0, \infty)$ such that

$$
|K(t, s, x)-K(t, s, y)| \leq G(t, s) \sqrt{\frac{(x-y)^{4}}{1+(x-y)^{2}}}
$$

for all $s, t \in[0, T]$ and $x, y \in \mathbb{R}$ with $x \geq y$,

(iv) $\sup _{t \in[0, T]} \int_{0}^{T} G(t, s)^{2} d s \leq \frac{1}{T}$.

Then the integral (8) has a solution $u^{*} \in C[0, T]$.

Proof We, first, define $T: C[0, T] \rightarrow C[0, T]$ by

$$
T u(t)=\int_{0}^{T} K(t, s, u(s)) d s+g(t), \quad t \in[0, T], u \in C[0, T] .
$$

We first prove that $T$ is nondecreasing. Assume that $v \preceq u$. From (ii), for all $s, t \in[0, T]$, we have $K(t, s, v(s)) \leq K(t, s, u(s))$. Thus, we get

$$
\begin{aligned}
\operatorname{Tv}(t) & =\int_{0}^{T} K(t, s, v(s)) d s+g(t) \\
& \leq \int_{0}^{T} K(t, s, u(s)) d s+g(t)=T u(t) .
\end{aligned}
$$

Now, for all $u, v \in C[0, T]$ with $v \preceq u$, due to (iii), we derive that

$$
\begin{aligned}
|T u(t)-T \nu(t)| & \leq \int_{0}^{T}|K(t, s, u(s))-K(t, s, v(s))| d s \\
& \leq \int_{0}^{T} G(t, s) \sqrt{\frac{(u(s)-v(s))^{4}}{(u(s)-v(s))^{2}+1}} d s .
\end{aligned}
$$

On account of the Cauchy-Schwarz inequality in the last integral above, we find that

$$
\begin{aligned}
& \int_{0}^{T} G(t, s) \sqrt{\frac{(u(s)-v(s))^{4}}{(u(s)-v(s))^{2}+1}} d s \\
& \quad \leq\left(\int_{0}^{T} G(t, s)^{2} d s\right)^{\frac{1}{2}}\left(\int_{0}^{T}\left(\sqrt{\frac{(u(s)-v(s))^{4}}{(u(s)-v(s))^{2}+1}}\right)^{2} d s\right)^{\frac{1}{2}} .
\end{aligned}
$$

Taking (iv) into account, we estimate the first integral in (9) as follows:

$$
\left(\int_{0}^{T} G(t, s)^{2} d s\right)^{\frac{1}{2}} \leq \frac{1}{\sqrt{T}}
$$


For the second integral in (9) we proceed in the following way:

$$
\left(\int_{0}^{T} \frac{(u(s)-v(s))^{4}}{(u(s)-v(s))^{2}+1} d s\right)^{\frac{1}{2}} \leq \sqrt{T} \sqrt{\frac{(d(u, v))^{4}}{(d(u, v))^{2}+1}} .
$$

By combining the above estimation, we conclude that

$$
|T u(t)-T v(t)| \leq \sqrt{\frac{(d(u, v))^{4}}{(d(u, v))^{2}+1}} .
$$

It yields

$$
d(T u, T v) \leq \sqrt{\frac{(d(u, v))^{4}}{(d(u, v))^{2}+1}},
$$

or equivalently,

$$
(d(T u, T v))^{2} \leq \frac{(d(u, v))^{4}}{(d(u, v))^{2}+1} .
$$

Now, if choose $\varphi(t)=t^{2}$ and $\psi(t)=t^{2}-\frac{t^{2}}{1+t^{2}}$, then we get

$$
\varphi(d(T u, T v)) \leq \varphi(d(u, v))-\psi(d(u, v))
$$

for all $u, v \in C[0, T]$ with $v \preceq u$. Hence, all hypotheses of Corollary 11 are satisfied. Thus, $T$ has a fixed point $u^{*} \in C[0, T]$ which is a solution of (8).

\section{Competing interests}

The authors declare that they have no competing interests.

\section{Authors' contributions}

All authors contributed equally and significantly in writing this article. All authors read and approved the final manuscript.

\section{Author details}

'Department of Mathematics, Texas A\&M University-Kingsville, Kingsville, 78363-8202, USA. ${ }^{2}$ King Abdulaziz University, Jeddah, 21589, Saudi Arabia. ${ }^{3}$ Department of Mathematics, Atilim University, Incek, Ankara 06836, Turkey. ${ }^{4}$ Nonlinear Analysis and Applied Mathematics Research Group (NAAM), King Abdulaziz University, Jeddah, 21589, Saudi Arabia.

${ }^{5}$ Department of Mathematics, University of Jaén, Campus Las Lagunillas, s/n, Jaén, 23071, Spain.

\section{Acknowledgements}

Antonio-Francisco Roldán-López-de-Hierro has been partially supported by Junta de Andalucía by project FQM-268 of the Andalusian CICYE.

Received: 14 August 2014 Accepted: 4 December 2014 Published: 22 Dec 2014

\section{References}

1. Roldán, A, Martínez-Moreno, J, Roldán, C: Multidimensional fixed point theorems in partially ordered metric spaces. J. Math. Anal. Appl. 396, 536-545 (2012)

2. Karapınar, E, Roldán, A, Martínez-Moreno, J, Roldán, C: Meir-Keeler type multidimensional fixed point theorems in partially ordered metric spaces. Abstr. Appl. Anal. 2013, Article ID 406026 (2013)

3. Roldán, A, Martínez-Moreno, J, Roldán, C, Karapınar, E: Multidimensional fixed-point theorems in partially ordered complete partial metric spaces under $(\psi, \varphi)$-contractivity conditions. Abstr. Appl. Anal. 2013, Article ID 634371 (2013)

4. Wang, S: Multidimensional fixed point theorems for isotone mappings in partially ordered metric spaces. Fixed Point Theory Appl. 2014, 137 (2014) 
5. Radenović, S, Kadelburg, Z, Jandrlić, D, Jandrlić, A: Some results on weakly contractive maps. Bull. Iran. Math. Soc. 38(3), 625-645 (2012)

6. Karapınar, E: Fixed point theory for cyclic weak $\phi$-contraction. Appl. Math. Lett. 24(6), 822-825 (2011)

7. Popescu, O: Fixed points for $(\psi, \phi)$-weak contractions. Appl. Math. Lett. 24(1), 1-4 (2011)

8. Zhang, Q, Song, Y: Fixed point theory for generalized $\phi$-weak contractions. Appl. Math. Lett. 22, 75-78 (2009)

9. Rouhani, $\mathrm{BD}$, Moradi, S: Common fixed point of multivalued generalized $(\psi, \phi)$-weak contractive mappings. Fixed Point Theory Appl. 2010, Article ID 708984 (2010)

10. Harjani, J, Sadarangani, K: Generalized contractions in partially ordered metric spaces and applications to ordinary differential equations. Nonlinear Anal. 72(3-4), 1188-1197 (2010)

11. Nieto, JJ, Pouso, RL, Rodríguez-Lopez, R: Fixed point theorems in partially ordered sets. Proc. Am. Math. Soc. 132(8), 2505-2517 (2007)

10.1186/1687-1812-2014-245

Cite this article as: Agarwal et al.: Some remarks on 'Multidimensional fixed point theorems for isotone mappings in partially ordered metric spaces'. Fixed Point Theory and Applications 2014, 2014:245

Submit your manuscript to a SpringerOpen ${ }^{\circ}$ journal and benefit from:

- Convenient online submission

- Rigorous peer review

- Immediate publication on acceptance

- Open access: articles freely available online

- High visibility within the field

- Retaining the copyright to your article

Submit your next manuscript at $\gg$ springeropen.com 